\title{
ESTUDO DA FREQÜÊNCIA DE OVOS DE HELMINTOS INTESTINAIS EM SANITÁRIOS DE USO PÚBLICO DE SOROCABA, SP
}

\author{
Tuffi Aidar Sobrinho, Lina Maria De Petrini da Silva Coelho, Sônia Maria de \\ Oliveira, João de Toledo Martins, José Augusto Rabello Júnior, Cláudia \\ Renata Paes de Oliveira, Maurício Aguiar de Paula, Maurício Wesley Perroud \\ Júnior e Sebastião Mitsuji Miyazaki
}

\begin{abstract}
Foram pesquisados 405 sanitários sendo 11 de praças, 2 da estação ferroviária, 4 da estação rodoviária, 55 de bares e restaurantes, 146 de escolas estaduais, 116 de escolas municipais, 8 de escolas particulares, 36 de postos de saude, 16 de centros esportivos, 8 de orfanatos e 3 de shopping. Foram usadas lâminas de microscopia com fita adesiva. O material foi coletado pela colagem da fita nos seguintes elementos: 4 campos do assento, maçanetas interna e externa, trinco, registro de torneira e descarga (botão e puxador). Dos 405 sanitários pesquisados, 22 (5,43\%) estavam contaminados. Foram encontrados ovos de: Ascaris lumbricoides, ancilostomideos, Enterobius vermicularis, Taenia spe Hymenolepis nana, sendo que em 2 sanitários foram encontrados, concomitantemente, ovos de 2 parasitas.
\end{abstract}

Palavras-chaves: Sanitário. Ovos de enteroparasitas. Helmintoses intestinais.

Dos diversos problemas de saúde pública que atingem a população brasileira, devido às baixas condições sócio-econômico-culturais que esta apresenta, é de notória importância a alta incidência de infecção por parasitas intestinais. Muitas vezes esta ocorre como único processo mórbido do paciente, ocasionando intensos distúrbios orgânicos e em outras vem agravar patologias concomitantes ${ }^{2}$.

Estudos de âmbito nacional demonstraram que o Estado de São Paulo, o mais desenvolvido da federação, apresentou cerca de $34,4 \%$ de infectados com enteroparasitas, enquanto o Estado da Bahia apresentou $84,1 \%$ de infectados, com média nacional de $55,3 \%$, fato que levou os autores a relacionar enfaticamente o nível sócio - econômico às taxas de infecção ${ }^{3}$.

No município de Sorocaba, SP, encontrou-se em população de pré-escolares uma incidência de parasitoses intestinais que variou entre $21,9 \%$ e $57,7 \%$, sendo observada a relação dessa incidência com hábitos de não lavagem das mãos antes das

\footnotetext{
Centro de Ciências Médicas e Biológicas da Pontificia Universidade Católica de São Paulo, Sorocaba, SP.

Órgão financiador: Conselho de Ensino e Pesquisa da PUCSP. Endereço para correspondência: Dr.Tuffi Aidar Sobrinho. Caixa Postal 160, 18001-970 Sorocaba, SP.

Recebido para publicação em 20/04/94.
}

refeições, de levar as mãos e objetos à boca e presença de unhas sujas ${ }^{14}$.

$\mathrm{O}$ indivíduo parasitado, pelos seus dejetos, contamina seu próprio ambiente com cistos e ovos de parasitas intestinais; a água pode acumulá-los e transportá-los a grandes distâncias; e os maus hábitos higiênicos citados acima e a ingestão de alimentos crus contaminados os levam ao hospedeiro suscetível ${ }^{6}$.

As principais helmintoses intestinais em relação à sua incidência são: a ascaridíase, a tricuríase, a ancilostomíase, a enterobíase, a estrongiloidíase, a himenolepíase e a teníase 3456 .

A pesquisa de Rubens Campos e cols, feita em 1988 , mostrou que na população brasileira $56 \%$ dos indivíduos parasitados eram do sexo masculino e $54 \%$ do sexo feminino ${ }^{3}$.

Em vista destes dados da literatura, surgiu a idéia de pesquisar a presença de ovos de enteroparasitas nos elementos dos sanitários manuseados pelos usuários, procurando relacionálos.

Para atingir este objetivo foram investigados os elementos dos sanitários de uso público de Sorocaba. Foram relacionados os sanítários segundo à sua localização, tipo de usuário quanto ao sexo e tipo de estabelecimento. 
Sobrinho TA, Coelho LMDPS, Oliveira SM, Martins JT, Rabello Júnior JA, CRP Oliveira, Paula MA, Perroud Júnior MW, Miyazak SM. Estudo da freqüência de ovos de helmintos intestinais em sanitários de uso público de Sorocaba, SP. Revista da Sociedade Brasileira de Medicina Tropical 28:33-37, jan-mar, 1995.

Cabe salientar que nenhum trabalho foi encontrado na literatura diretamente relacionado a este.

\section{MATERIAL E MÉTODOS}

Foi coletado material nos sanitários dos logradouros públicos da cidade de Sorocaba, SP, tais como: escolas estaduais, municipais e particulares; estações ferroviária e rodoviária; postos de saúde; centros esportivos; praças; orfanatos; bares; restaurantes e shopping center. Foram pesquisados todos os logradouros de cada uma das modalidades acima citadas, exceto os bares e restaurantes.

Foram pesquisados 405 sanitários sendo 181 masculinos, 175 femininos e 49 de uso comum, pertencentes a 81 bairros. O material foi coletado de 8 sanitários de 4 escolas particulares, 116 sanitários de 58 escolas municipais, 146 sanitários de 73 escolas estaduais, 2 sanitários da estação ferroviária, 4 da estação rodoviária, 11 sanitários de 6 praças, 16 sanitários de 8 centros esportivos, 36 sanitários de 18 centros de saúde, 8 sanitários de 2 orfanatos e 55 sanitários de 51 bares e restaurantes, e 3 sanitários de shopping center.

$\mathrm{O}$ método de colheita de material foi o da fita adesiva transparente sobre lậmina de vidro. Este consiste na montagem de lâmina de vidro com uma tira de $4 \mathrm{~cm}$ de comprimento de fita adesiva colada longitudinalmente sobre ela, com uma ponta dobrada. Esta fita foi aplicada à superfície de cada elemento do sanitário pesquisado e posteriormente recolada sobre a lâmina. Para a identificação da lâmina usou-se etiqueta colada a uma de suas extremidades, contendo um número referente ao sanítário pesquisado, uma letra minúscula referente ao sexo dos usuários e uma letra maiúscula referente ao elemento do sanitário (Figura 1).

a

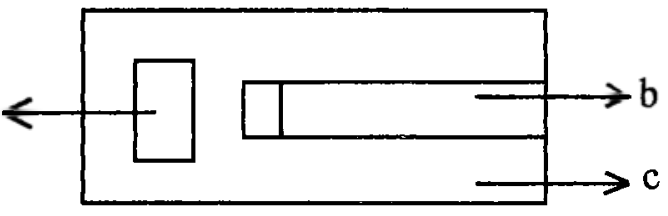

Figura 1 - Lâmina montada para coleta de material: $a$. etiqueta; $b$. fita adesiva transparente; c. lâmina de vidro.
De cada sanitário foram investigados os seguintes elementos: assento do vaso(subdividido em quadrantes), maçanetas interna e externa, trinco, descarga (botão ou puxador) e registro da torneira.

Após a colheita das amostras as lâminas foram lidas, diretamente, em microscopia óptica comum com os aumentos de 100 e 400 vezes. Os resultados da leitura foram armazenados em fichas individuais para cada sanitário, com as seguintes informações: tipo de logradouro, endereço, sexo dos usuários, elementos pesquisados.

Posteriormente foi feita a tabulação dos resultados em fichas, agrupando-os conforme o sexo dos usuários, natureza e localização do logradouro onde o sanitário estava situado, elementos do sanitário e identificação do enteroparasita encontrado.

As lâminas que possuiam enteroparasitas foram documentadas por fotografação em microscopia óptica comum.

\section{RESULTADOS}

Dos 405 sanitários pesquisados $22(5,43 \%)$ apresentaram ovos de enteroparasitas.

Foram encontrados ovos de Ascrris lumbricoides 18 vezes, de ancilostomídeos 2 vezes, de Enterobius vermicularis 2 vezes, de Taenia sp e Hymenolepis nana apenas uma vez (Figuras 2, 3, 4 e 5).

Nos logradouros onde foi efetuada a pesquisa foram obtidos os seguintes resultados: dos 2 sanitários da estação ferroviária 1 (50\%) estava contaminado; $3(37,5 \%)$ em 8 de orfanatos; 5

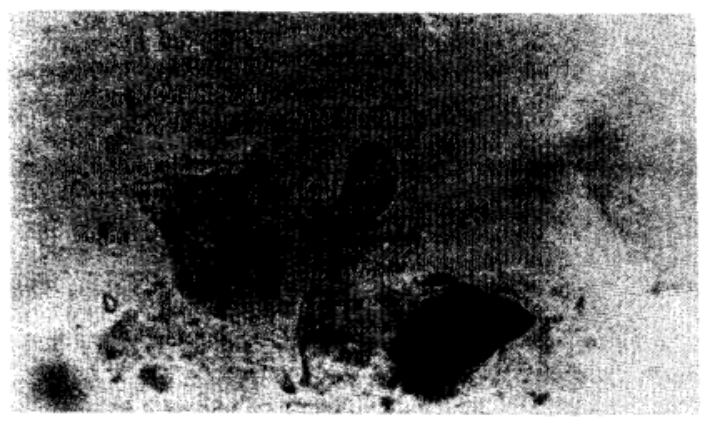

Figura 2 - Fotomicrografia óptica de ovo de Ascaris lumbricoides. $480 X$. 
Sobrinho TA, Coelho LMDPS, Oliveira SM, Martins JT, Rabello Júnior JA, CRP Oliveira, Paula MA, Perroud Júnior MW, Miyazak SM. Estudo da freqüência de ovos de helmintos intestinais em sanitários de uso público de Sorocaba, SP. Revista da Sociedade Brasileira de Medicina Tropical 28:33-37, jan-mar, 1995.

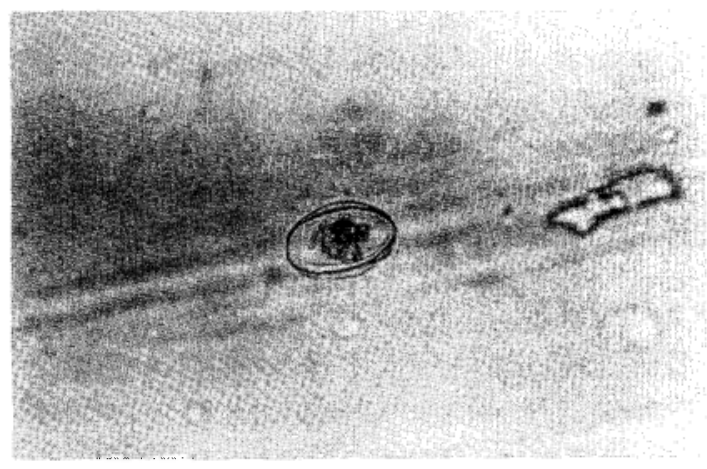

Figura 3 - Fotomicrografia óptica de ovo de ancilostomídeos. $480 \mathrm{X}$.

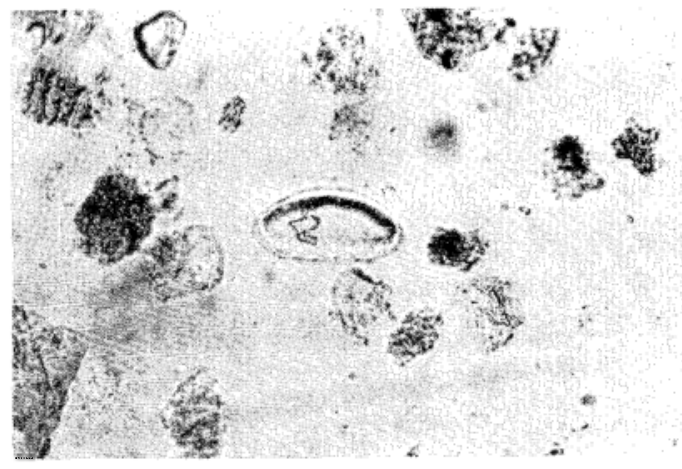

Figura 4 - Fotomicrografia óptica de ovo de Enterobius vermiculares. $480 X$.

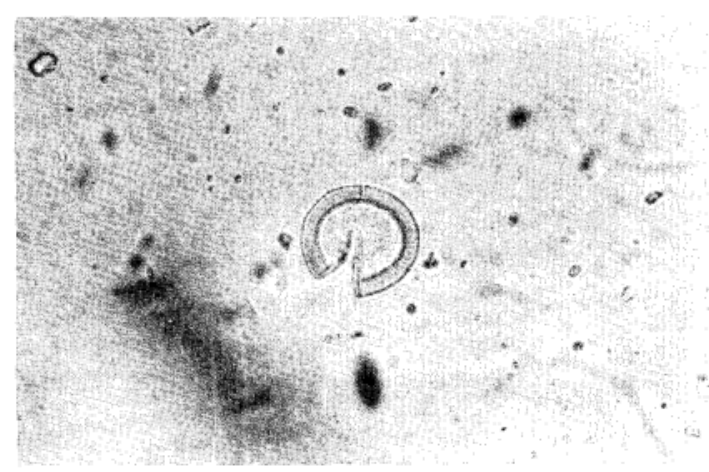

Figura 5 - Fotomicrografia óptica de ovo de Taenia sp. $480 X$.

$(9,09 \%)$ em 55 de bares e restaurantes; $1(9,09)$ em 11 sanitários de praças; $11(4,07 \%)$ em 270 sanitários de escolas; 1 (2,78\%) em 36 sanitários dos postos de saúde e nenhum nos da estação rodoviária, dos centros esportivos e do shopping center.
Relacionando o número de sanitários onde foram encontrados os referidos ovos com o número total de sanitários, os resultados foram: de bares e restaurantes $1,23 \%$, das escolas municipais $1,23 \%$, das escolas estaduais $0,99 \%$, de escolas particulares $0,49 \%$, de orfanatos $0,74 \%$, da estação ferroviária $0,25 \%$, de praças $0,25 \%$, dos postos de saúde $0,25 \%$ (Tabela 1 ).

Tabela 1 - Sanitários examinados distribuídos por logradouros.

\begin{tabular}{lrcc}
\hline Logradouros & $\begin{array}{c}\text { Sanitários } \\
\text { examinados }\end{array}$ & \multicolumn{2}{c}{$\begin{array}{c}\text { Sanitários } \\
\text { contaminados }\end{array}$} \\
\cline { 2 - 4 } & & $\mathrm{n}^{\mathbf{0}}$ & $\%$ \\
\hline Bares e restaurantes & 55 & 5 & 1,23 \\
Escolas municipais & 116 & 5 & 1,23 \\
Escolas estaduais & 146 & 4 & 0,99 \\
Escolas particulares & 8 & 2 & 0,49 \\
Orfanatos & 8 & 3 & 0,74 \\
Estação ferroviária & 2 & 1 & 0,25 \\
Praças & 11 & 1 & 0,25 \\
Postos de saúde & 36 & 1 & 0,25 \\
Estação rodoviária & 4 & 0 & 0,00 \\
Centros esportivos & 16 & 0 & 0,00 \\
Shopping center & 3 & 0 & 0,00 \\
\hline Totais & 405 & 22 & 5,43 \\
\hline
\end{tabular}

Nos sanitários pesquisados nas escolas os resultados foram: dos 8 das escolas particulares 2 (25\%) apresentaram ovos de helmintos, dos 116 das municipais $5(4,31 \%)$ mostraram a presença de ovos de helmintos, dos 146 das estaduais $4(2,74 \%)$ os apresentaram (Tabela 2). Em relação ao total de sanitários pesquisados a frequência foi de $0,49 \%$ nas escolas particulares, $1,23 \%$ nas escolas municipais e de $0,99 \%$ nas estaduais.

Tabela 2 - Contaminação dos sanitários em escolas.

\begin{tabular}{lccc}
\hline Tipos de escolas & $\begin{array}{c}\text { Sanitários } \\
\text { pesquisados }\end{array}$ & $\begin{array}{c}\text { Sanitários } \\
\text { contami } \\
\text { nados }\end{array}$ & $\begin{array}{c}\text { \% de } \\
\text { contami- } \\
\text { nação }\end{array}$ \\
\hline Particulares & 8 & 2 & 25,00 \\
Municipais & 116 & 5 & 4,31 \\
Estaduais & 146 & 4 & 2,74 \\
\hline
\end{tabular}


Sobrinho TA, Coelho LMDPS, Oliveira SM, Martins JT, Rabello Júnior JA, CRP Oliveira, Paula MA, Perroud Júnior MW, Miyazak SM. Estudo da frequiencia de ovos de helmintos intestinais em sanitários de uso público de Sorocaba, SP. Revista da Sociedade Brasileira de Medicina Tropical 28:33-37, jan-mar, 1995.

Foram pesquisados 81 bairros da cidade tendo sido encontrados 13 com sanitários contaminados com ovos de helmintos, o que corresponde a $16,0 \%$. Deste resultado, o centro da cidade foi responsável por $9,88 \%$ da contaminação com 8 sanitários apresentando ovos de enteroparasitas.

Em relação aos elementos dos sanitários pesquisados os resultados foram: de 405 assentos 21 estavam com ovos de hemintos correspondendo a 5,18\%; das 262 maçanetas internas 1 estava com os referidos ovos perfazendo $0,38 \%$; dos 389 trincos 4 os apresentaram , ou seja, 1,03\% de contaminação; e, em 353 botões de descarga 1 apresentou contaminação, sendo $0,28 \%$ (Tabela 3 ). No assento o quadrante posterior apresentou-se 10 vezes contaminado com ovos de helmintos, o anterior 5 vezes, o esquerdo 4 e odireito 2 vezes. Os percentuais de contaminação com ovos de enteroparasitas nos quadrantes do assento foram de $47,62 \%$ no posterior, $23,81 \%$ no anterior, $19,05 \%$ no esquerdo e de $9,52 \%$ no direito.

Os números e percentuais de contaminação, com ovos de helmintos nos sanitários, conforme o sexo dos usuários são os seguintes: dos 181 sanitários masculinos $11(6,08 \%)$ estavam contaminados; dos 175 femininos $6(3,43 \%)$ estavam contaminados; dos 49 de uso comum $5(10,20 \%)$ apresentaram contaminação (Tabela 4).

Tabela 3 - Contaminação dos elementos dos sanitários.

\begin{tabular}{lccr}
\hline $\begin{array}{l}\text { Elementos dos } \\
\text { sanitários }\end{array}$ & $\begin{array}{c}\mathrm{N}^{\mathbf{0}} \text { de } \\
\text { contaminados }\end{array}$ & $\begin{array}{c}\text { \% de } \\
\text { contaminação }\end{array}$ & Total \\
\hline Assento & 21 & 5,18 & 405 \\
Trinco & 4 & 1,03 & 389 \\
Maçaneta interna & 1 & 0,38 & 262 \\
Descarga - botão & 1 & 0,28 & 353 \\
Descarga - puxador & 0 & 0,00 & 52 \\
Maçaneta externa & 0 & 0,00 & 262 \\
Registro - torneira & 0 & 0,00 & 399 \\
\hline
\end{tabular}

Tabela 4 - Contaminação com relação ao sexo do usuário.

\begin{tabular}{lccc}
\hline Sexo do usuário & $\begin{array}{c}\text { Sanitários } \\
\text { pesquisados }\end{array}$ & $\begin{array}{c}\text { Sanitários } \\
\text { contami- } \\
\text { nados }\end{array}$ & $\begin{array}{c}\% \text { de } \\
\text { contami- } \\
\text { nação }\end{array}$ \\
\hline Feminino & 175 & 6 & 3,43 \\
Masculino & 181 & 11 & 6,08 \\
Ambos & 49 & 5 & 10,20 \\
\hline
\end{tabular}

\section{DISCUSSÃO}

A população brasileira em geral e a sorocabana em particular apresentam altos índices de infecção por enteroparasitas ${ }^{2} 34$. Isto favorece a contaminação do seu próprio ambiente com os ovos dos mesmos ${ }^{6}$. Os elementos dos sanitários manuseados pelos usuários seriam, portanto, suscetíveis de contaminação, o que gerou esta pesquisa.

Os resultados nela obtidos comprovaram esta suspeita. Entretanto, o percentual de contaminação comovos de hel mintos obtido no estudo dos sanitários apresentou-se relativamente baixo $5,43 \%$, enquanto estudos anteriores mostraram uma frequência variando de $21,90 \%$ a $57,70 \%$ em crianças sorocabanas $^{14}$.

Dentre os bairros com sanitários contaminados, o centro apresentou maior percentual de contaminação. Isto provavelmente decorre da alta rotatividade da população usuária dos sanitários do centro de Sorocaba, e que aflui de todos os bairros.

Os sanitários usados pela população masculina apresentaram maior incidência de ovos de helmintos que os de uso feminino sendo a diferença de 2,55\%, confirmando dado da literatura que cita $2,00 \%$ a mais na incidência de parasitoses intestinais na população masculina ${ }^{3}$. Nos sanitários de uso comum houve um percentual de contaminação ainda maior que os masculinos e femininos, $10,20 \%$. Os sanitários de uso comum eram de bares erestaurantes. É importante salientar que bares e restaurantes de bairros periféricos não tinham qualquer espécie de sanitário, fato que deve ter contribuido para o percentual maior de contaminação nos sanitários do centro.

Os postos de saúde, apesar da frequência de população menos favorecida, apresentam baixo indice de contaminação, apenas 1 sanitário dos 18 postos. Estes dados se contrapõem aos da estação ferroviária e dos orfanatos, que apresentaram os dois maiores percentuais de sanitários contaminados, embora com o mesmo tipo de população usuária.

$\mathrm{Na}$ pesquisa dos sanitários das escolas os resultados seapresentaram dissonantes da expectativa resultante dos dados da literatura, pois, esta relaciona maior incidência de enteroparasitoses com 
Sobrinho TA, Coelho LMDPS, Oliveira SM, Martins JT, Rabello Júnior JA, CRP Oliveira, Paula MA, Perroud Júnior MW, Miyazak SM. Estudo da freqüência de ovos de helmintos intestinais em sanitários de uso público de Sorocaba, SP. Revista da Sociedade Brasileira de Medicina Tropical 28:33-37, jan-mar, 1995.

populações de baixas condições sócio-econômicoculturais ${ }^{2}$ e nas escolas particulares o percentual de sanitários contaminados foi bem maior que nas públicas (municipais e estaduais) (Tabela 2). Não se pode, entretanto, afirmar que este resultado indique que as respectivas populações usuárias tenham proporcional índice de infecção.

Dos elementos dos sanitários contaminados o assento apresentou maior ocorrência de ovos de helmintos, principalmente no seu quadrante posterior, o que correspondeu às expectativas naturais devido ao uso.Nos demais elementos os mais manuseados foram também os de maior contaminação, embora com índices menores que o do assento.

Quanto aos tipos de enteroparasitas, cujos ovos foram detectados nos sanitários, a frequência maior de Ascaris lumbricoides concorda com o que a literatura afirma em relação à população' 346 . Entretanto, a diferença de frequência dos ovos deste parasita é muito maior que a dos demais nos sanitários que na população. Isto pode ser decorrente do tipo de envoltório de seu ovo, que sendo mamelonado propicia maior aderência às superfícies.

A frequência de ovos de enteroparasitas nos elementos dos sanitários de uso público da cidade de Sorocaba não serve de parâmetro para avaliar o indice de contaminação dos usuários, mas, apenas para detectar a sua ocorrência.
Sendo esta uma primeira pesquisa no gênero, inclusive quanto ao método de coleta, é possível que o percentual de contaminação obtido não corresponda fielmente à contaminação dos elementos dos sanítários. Muitos outros fatores podem também ter interferido nessa variação, tais como: as condições de higiene dos sanitários, o tipo de superfície dos ovos dos helmintos que lhes pode conferir maior ou menor aderência e a probabilidade de um indivíduo infectado não ser contaminador no momento do uso dos sanitários.

\section{SUMMARY}

Four hundredand five water closets were investigated: 11 located at public squares, 2 at the railroad station, 4 at the bus station, 55 at bars and restaurants, 146 at state schools, 116 at municipal schools, 8 at private schools, 36 at public health centers, 16 at sports centers, 8 at orphanages and 3 at shopping malls. Microscopy slides with adhesive tape were used. The material was obtained by sticking the tape onto the following elements: 4 spots on the toilet seat, internal and external door knobs, latch, faucet handle and discharge valve (push button or pulling string). Out of the 405 water closets studied, $22(5,43 \%)$ were contaminated. Eggs of Ascaris lumbricoides, Ancylostomatidae, Enterobius vermiculares, Taenia $s p$ and Hymenolepis nana were found. In 2 water closets eggs of 2 parasites were found simultaneoushy.

Key-words: Water closet. Sanitary. Eggs of enteroparasites. Intestinal helminthiasis.

\section{REFERÊNCIAS BIBLIOGRÁFICAS}

1. Aidar Sobrinho T, Coelho LMPS, Oliveira SM, Chiaparini LC. Prevalência de parasitoses intestinais em pré escolares da rede municipal de ensino de Sorocaba. In: Resumos do XI Congresso Brasileiro de Parasitologia, Rio de Janeiro p.69, 1989

2. Amato Neto V. Atualização-Parasitoses intestinais: apresentação. Clínica Pediátrica 1:25, 1977.

3. Campos R, Briques W, Belda Neto M, Souza JM, KatzN, Salata E, Dacal ARG, Dourado H, Castanho REP, Gurvitz R, Zingano A, Pereira GJM, Ferriolli Filho F, Camillo-Coura L, Faria JAS, Cimerman B, Siqueira Filho JB, Prata A. Levantamento multicêntrico de parasitoses intestinais no Brasil. Rhodia - grupo Rhône- Poulen, 1988.
4. Duarte LR. Incidência de parasitoses intestinais em pré-escolares no município de Sorocaba, São Paulo. Tese de mestrado, Pontifícia Universidade Católica de São Paulo, Sorocaba, 1985.

5. Guido GL, Amato Neto V. Parasitoses intestinais: incidência e transmissão. Clínica Pediátrica 1:2628,1977.

6. Marzochi MCA, CarvalheiroJR. Estudos dos fatores envolvidos na disseminação dos enteroparasitas. III- Distribuição de alguns enteroparasitas em dois grupos populacionais da cidade de Ribeirão Preto, São Paulo, Brasil. Revista do Instituto de Medicina Tropical de São Paulo 20:31-35, 1978. 